\title{
Designing Menus for Multilevel Demand Subscription
}

\author{
Yuting Mou, Céline Gérard, Anthony Papavasiliou, Philippe Chevalier \\ CORE, LIDAM, UCLouvain \\ \{yuting.mou, celine.gerard, anthony.papavasiliou, philippe.chevalier\}@uclouvain.be
}

\begin{abstract}
Priority service and its generalization, multilevel demand subscription, have been proposed in the literature as alternatives to real-time pricing in residential demand response aggregation. This paper proposes a modeling approach for designing multilevel demand subscription menus that are comparable to priority service pricing menus. The menus are designed as the equilibrium solution to a Stackelberg game, which is modeled as a bilevel optimization problem involving a vertically integrated utility and consumers. The problem is reformulated and solved as a mixed integer linear program. The menus are compared in a realistic model of the Belgian power market.
\end{abstract}

\section{Introduction}

Despite advances in information and communication technology which support the deployment of demand response, real-time pricing has encountered challenges in fully delivering its promise for mobilizing demand-side flexibility $[1,2]$. To a certain extent, this is due to the fact that residential consumers do not have sufficient financial incentives or attention bandwidth for engaging in the real-time trading of electricity. This has motivated the development of residential demand response aggregation paradigms based on the premise that households view electricity as a service with differentiated quality $[3,4,5,6]$. Inspired by successful examples of quality differentiation in other deregulated industries, these alternative demand response paradigms aim at striking a balance between economic efficiency and implementation complexity.

Priority service [7] corresponds to the most basic variation of quality differentiation. Priority service differentiates electricity supply according to reliability, with higher reliability corresponding to a higher price. In this setting of asymmetric information, households are characterized by a privately known valuation that the aggregator does not have access to. The aggregator is then tasked with designing a menu of services, such that consumers self-select reliability levels that are consistent with the reliability that the inherently stochastic supply mix of the aggregator can deliver. Chao [7] demonstrates how an aggregator can design such an optimal menu by relying on an estimate of the system demand function, which is equivalent to a distribution of consumer types. Other approaches in the literature use quality differentiation along different dimensions, for example the deadline of delivering a certain amount of energy $[8,9,10]$ in the case of deferrable demand.

Multilevel demand subscription [11] generalizes this setting by allowing the aggregator to differentiate service along reliability and duration. The challenge of information asymmetry remains, with consumer types now characterized by a privately known index corresponding to their position on the system load duration curve. The aggregator uses this information in order to design a service offering that differentiates prices as a function of both reliability and duration. Note that after subscribing to a service offering (for multilevel demand subscription or for priority service), the consumer can be surveyed by a utility through customer satisfaction surveys. This creates the possibility for the utility to improve the design of the menu through iterative communication with consumers and adaption of the offered menu.

Whereas the aforementioned theory of quality differentiation [7, 11] is a valuable starting point for investigating alternatives to real-time pricing, it is limited to analytical models that rely on stringent assumptions (e.g. synchronization of loads, idealized and abstract representations of households). We provide two main contributions in this work. (i) Inspired by recent research on priority service [5], we cast the multilevel demand subscription menu design problem of Chao [11] as a Stackelberg game, which we express equivalently as a mixed integer linear program. For this purpose, we define a mapping from time sorted in a load duration curve to real time. We propose a fuse limit 
model in the spirit of Margellos [4] in order to compute demand functions for individual types of households. In order to accommodate non-synchronous loads, we approximate the resulting system demand function, in order to arrive to concave system demand function that allows us to apply the standard menu design theory [11]. (ii) We use this reformulation in order to perform a case study that compares the two paradigms on eight types of households in a realistic model of the Belgian market. We use our reformulation in order to represent complex households with distributed storage and local stochastic renewable supply, thereby extending the original theory $[7,11]$ to a realistic modeling setting.

\section{Overview of Priority Service and Multilevel Demand Subscription}

\subsection{Priority Service}

In priority service, electricity is considered as a service with different levels of quality. The feature of service that differentiates quality is reliability. Concretely, an aggregator proposes a set of options for procuring capacity strips. Each option is characterized by a different level of reliability. More reliable options are more expensive to procure. In this work, we consider three reliability differentiated options following the ColorPower concept [6]. Based on the offered menu, the household subscribes to a particular amount of capacity for each option, and enrolls in a long term (e.g. annual) contract for electricity service. Once a household is subscribed, a home energy router allocates particular devices within the house to strips of different color by ensuring that the mean power within an interval under a certain option does not exceed the subscribed amount of kilowatts for that particular color.

In offering priority service contracts to residential consumers, the utility commits to a certain level of reliability for each service option. This level of reliability must be respected on average over an extended period of service (e.g. annually) even if certain periods of service may be characterized by fluctuations around this average [5]. In a central dispatch setting, the utility interrupts colors in order of decreasing reliability, since the menu is designed so that higher-valuation consumers self-select higher levels of reliability. The task of the utility is to design the menus carefully, in order to ensure that households are segmented adequately. In doing so, the information asymmetry challenge is that the aggregator is not aware of the valuation of each individual consumer for power, as this is private information. The utility can only estimate aggregate statistical information about the consumer population $[5,7]$ in the form of demand functions.

\subsection{Multilevel Demand Subscription}

Multilevel demand subscription generalizes the priority service model of the previous section. From the point of view of consumers, kilowatts of different priority levels (i.e. colors) are "topped up" with credits. More credits entitle customers to use more hours of power of a certain quality, but cost more. The proposed contract is a forward contract. This implies that there is an inherent "override" option (which is activated automatically when the customer exceeds the capacity and duration limits of the contract), however in that case the customer would need to procure any additional consumption at the prevailing real-time price. Thus, the energy router within the home needs to respect not only the power limit of each color, but also the total number of credits over the service period (e.g. over the week or day). Clearly, this service offering presents increased complexity from the point of view of the household. However, it also allows the utility to better discriminate among consumer types. This contributes towards more efficient allocation of power to flexible demand.

Accordingly, the utility commits not only to honoring the reliability of each service option, but also to honoring the duration of that option. The task of pricing the menu also becomes more challenging. Although the utility can still rely on aggregate statistical information about the population, it is now required to estimate a load duration curve for the population, parametric on a retail price.

\section{Characterizing Household Types}

As we explain in section 2.2 , the utility must quantify the valuation of an increment of power for a certain duration in order to design the multilevel demand subscription menu that is offered to consumers. The idea of our proposed approach is to estimate the marginal value of an increment in the fuse limit of a household. However, due to the coexistence of storage and batteries in a household, there is no guarantee that this marginal value is a concave function of the duration of consumption. Therefore, in a second phase, we compute the closest concave approximation of this estimate. We use this function as input to the menu design problem of section 4 .

In order to estimate the valuation of an increment of power for a certain duration, we propose a stochastic optimization program for each type of household. This mathematical program allows us to represent battery and load shedding decisions over a day, when we enforce a fuse limit on the household that is equipped with 
solar panels and must serve a mix of flexible and inflexible load. We model two types of uncertainty, long-term and short-term. Long-term uncertainty is represented by a set of scenarios that correspond to seasonal variations. In the case study of section 6 , the set $\mathcal{S}$ consists of weekdays and weekends for each season of the year. Each scenario $s \in \mathcal{S}$ occurs with a probability $P_{s}$. For each scenario, short-term uncertainty, which corresponds to the real-time production of solar panels, is modeled by a scenario tree with outcomes of solar panel production for each time stage of the day. We denote by $\omega_{[t]}^{s} \in \Omega_{[t]}^{s}$ the sequence of solar panel production up to stage $t$, while in scenario $s$.

We express the household model that we use for creating a system load duration curve as follows, for household type $h \in \mathcal{H}$ :

$$
\begin{aligned}
& \left(H V_{h}\right): \\
& \min _{\substack{l s, n d, e, b c, b d}} V_{c u t} \sum_{s \in \mathcal{S}} \sum_{t \in \mathcal{T}} \sum_{\omega_{[t]}^{s} \in \Omega_{[t]}^{s}} P_{s} P_{t, \omega_{[t]}^{s}}^{s} l s_{t, s, \omega_{[t]}^{s}} \\
& \text { s.t. } \quad 0 \leq b d_{t, s, \omega_{[t]}^{s}} \leq B D_{h}, \quad t \in \mathcal{T}, s \in \mathcal{S}, \\
& \omega_{[t]}^{s} \in \Omega_{[t]}^{s} \\
& 0 \leq b c_{t, s, \omega_{[t]}^{s}} \leq B C_{h}, \quad t \in \mathcal{T}, s \in \mathcal{S}, \\
& \omega_{[t]}^{s} \in \Omega_{[t]}^{s} \\
& 0 \leq e_{t, s, \omega_{[t]}^{s}} \leq E_{h}, \quad t \in \mathcal{T}, s \in \mathcal{S}, \\
& \omega_{[t]}^{s} \in \Omega_{[t]}^{s} \\
& e_{1, s, \omega_{1}^{s}}=-\frac{b d_{1, s, \omega_{1}^{s}}}{\eta_{h}^{d}}+b c_{1, s, \omega_{1}^{s}} \cdot \eta_{h}^{c}, \\
& s \in \mathcal{S}, \omega_{1}^{s} \in \Omega_{1}^{s} \\
& e_{t, s, \omega_{[t]}^{s}}=e_{t-1, s, A\left(\omega_{[t]}^{s}\right)}-\frac{b d_{t, s, \omega_{[t]}^{s}}}{\eta_{h}^{d}} \\
& +b c_{t, s, \omega_{[t]}^{s}} \cdot \eta_{h}^{c}, \quad t \in \mathcal{T} \backslash\{1\}, \\
& s \in \mathcal{S}, \omega_{[t]}^{s} \in \Omega_{[t]}^{s} \\
& D P_{t, s, h}-l s_{t, s, \omega_{[t]}^{s}}+b c_{t, s, \omega_{[t]}^{s}}-P V_{t, s, \omega_{t}^{s}} \\
& -b d_{t, s, \omega_{[t]}^{s}}=n d_{t, s, \omega_{[t]}^{s}}, \quad t \in \mathcal{T}, \\
& s \in \mathcal{S}, \omega_{[t]}^{s} \in \Omega_{[t]}^{s} \\
& \left(\lambda_{t, s, \omega_{[t]}^{s}}\right): \quad n d_{t, s, \omega_{[t]}^{s}} \leq F L, \quad t \in \mathcal{T}, s \in \mathcal{S}, \\
& \omega_{[t]}^{s} \in \Omega_{[t]}^{s} \\
& l s_{t, s, \omega_{[t]}^{s}} \geq 0, t \in \mathcal{T}, s \in \mathcal{S}, \omega_{[t]}^{s} \in \Omega_{[t]}^{s}
\end{aligned}
$$

The goal of the household is to minimize its economic damage from not serving part of its load. The parameter $V_{c u t}$ represents the risk-adjusted cost of consumers for accessing the spot market. Constraints (1), (2) and (3) represent, respectively, the discharge power, charge power and storage capacity limits of the household battery. Constraints (4) and (5) describe the dynamics of the battery, with $\eta_{h}^{c}$ and $\eta_{h}^{d}$ expressing respectively the charge and discharge efficiency of the household battery. The power balance in the household is represented by constraint (6) where parameter $D P_{t, s, h}$ corresponds to the inflexible load of household type $h$ at time $t$ for scenario $s$ and $P V_{t, s, \omega_{t}}$ is the solar panel production sample for that time stage. Finally, constraint (7) limits the power that can be drawn from the grid by the household using parameter $F L$ (i.e. the fuse limit of the household).

The dual multiplier $\lambda_{t, s, \omega[t]}$ of constraint (7) is used for quantifying the incremental value of the fuse limit. Concretely, the valuation for an additional unit of power in period $t$ of actual operations, given fuse limit $F L$, is computed as $\sum_{s \in \mathcal{S}} \sum_{\omega_{[t]} \in \Omega_{[t]}^{s}} \lambda_{t, s, \omega_{[t]}}, \quad t \in \mathcal{T}$. This valuation is then derived for different levels of fuse limit. We use this information in order to create a demand function for increments of power for each household type at a given operating interval.

\section{Multilevel Demand Subscription Menu Design and Reformulation as an MILP}

We now cast the multilevel demand subscription pricing problem as a bilevel optimization program. The problem has been formulated and solved in the literature as a Stackelberg equilibrium [11]. We depart from the classical description of the problem that is developed in [11], in order to allow for a more general representation of uncertainty and production constraints which are typically encountered in production simulation models. In this respect, we follow the approach described in [5], where the Stackelberg equilibrium formulation of priority service pricing [7] is cast as a bilevel optimization program. Bilevel formulations were already adopted in the literature to design other demand response contracts, e.g. in [12]. We reformulate the problem as a mixed integer linear program by exploiting its structure. The exposition here focuses on multilevel demand subscription pricing, with priority service pricing being a special case. The notation of each model is available in the electronic supplement, along with a toy example that emphasizes the value of multilevel demand subscription compared to priority service. ${ }^{1}$

\footnotetext{
${ }^{1}$ The electronic supplement is available at the following link: https://perso.uclouvain.be/anthony. papavasiliou/public_html/ElectronicAppendix_ HICSS2021_MDSPMenuDesign.pdf
} 


\subsection{Bilevel Mathematical Structure}

Chao [11] casts the multilevel demand subscription pricing problem as a Stackelberg equilirbium. The leader of this Stackelberg game is the utility that designs a multilevel demand subscription menu that is offered to consumers, who are the followers. Due to information asymmetry (see section 2.2), the leader integrates in the bilevel program the optimal reaction of the followers to the menu design problem. This gives rise to a mathematical program with equilibrium constraints that also integrates menu design with unit commitment. Note that, following the literature [11], we do not consider transmission or distributions constraints in our model. This bilevel model can be represented abstractly as follows, and is further illustrated in Figure 1.

$$
\begin{aligned}
\max _{\mathbf{m}, \mathbf{n}, \mathbf{o}, \mathbf{p}, \mathbf{r}, \boldsymbol{\pi}} & S W(\mathbf{m}, \mathbf{n}, \mathbf{o}, \mathbf{p}, \mathbf{d}, \mathbf{r}) \\
\text { s.t. } & (\mathbf{m}, \mathbf{n}, \mathbf{o}, \mathbf{p}, \mathbf{d}) \in \mathbf{X} \\
& \mathbf{r}=\phi\left(\mathbf{d}, \boldsymbol{\sigma}^{\star}\right) \\
& \boldsymbol{\sigma}^{\star} \in \arg \max _{\sigma}\{C S(\mathbf{r}, \boldsymbol{\pi}): \sigma \in \Sigma\}
\end{aligned}
$$

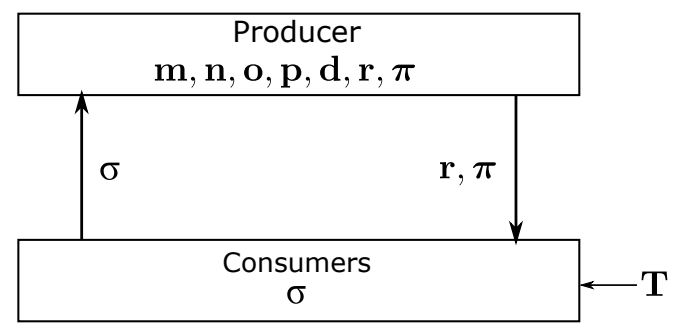

Figure 1. Interaction between the producer and consumers in the multilevel demand subscription bilevel model.

In this model, the variables $\mathbf{m}, \mathbf{n}, \mathbf{o}, \mathbf{p}$ correspond respectively to startup and shutdown decisions, unit commitment and power generation. The subscription quantity of each consumer to each option of the multilevel demand subscription menu is indicated by $\sigma$, while the supply to each option is indicated by $\mathbf{d}$. The reliability, duration and price of the options in the menu are denoted by $\mathbf{r}, \mathbf{T}$ and $\pi$ respectively. We assume that the duration is an exogenous parameter, and is therefore not a variable in the model.

The objective of the utility in this mathematical program is to maximize social welfare, as represented by function $S W$ in Eq. (9). The technical constraints of the producer are given in Eq. (10). Constraint (11) indicates that the designed price menu needs to deliver a promised level of reliability during a certain duration, which is influenced by the way consumers react to the offered menu. Finally, consumers decide on their subscription by maximizing their individual surplus (represented by the function $C S$ ), as shown by Eq. (12).

\subsection{Representation of the Household by the Utility}

From the point of view of the utility that is designing a service menu, the population of households can be represented by a distribution over valuations. This distribution is encoded in the parameters $\left(D_{l}, V_{l}(t)\right)$. The function $V_{l}(t)$ is a non-decreasing function of $t$, since more hours of consumption increase the benefit of the household. Note that the formulation of the consumer problem below implicitly assumes that $V_{l}(t)$ is further a concave function of $t$, otherwise it would be needed to sum over time periods in the first term of the objective function. Section 3 describes how the utility can estimate this function based on information about the distribution of installed equipment in residential households.

Given a set of consumer types $\mathcal{L}$, each of them as a follower subscribes to service options from a menu with $|\mathcal{I}| \cdot|\mathcal{J}|$ options. The set $\mathcal{I}$ expresses the offered reliability, while the set $\mathcal{J}$ includes the set of duration options. Each consumer type $l$ then solves the following problem for choosing an option from the menu, given the reliability $r_{i}$, duration $T_{j}$, and price $\pi_{i, j}$ of each option from the upper-level problem:

$$
\begin{aligned}
\left(C P_{l}\right): \max _{\boldsymbol{\sigma}_{l}} & \sum_{i \in \mathcal{I}, j \in \mathcal{J}} \sigma_{l, i, j}\left(r_{i} \cdot V_{l}\left(T_{j}\right)-\pi_{i, j}\right) \\
\text { s.t. } \quad & \sigma_{l, i, j} \geq 0, \quad i \in \mathcal{I}, j \in \mathcal{J} \\
\left(\gamma_{l}\right): & \sum_{i \in \mathcal{I}, j \in \mathcal{J}} \sigma_{l, i, j} \leq D_{l}
\end{aligned}
$$

The goal of the consumer is to maximize its profit from procuring an option $(i, j)$. The first term in the objective function of Eq. (13) is the expected benefit from procuring option $(i, j)$. The constraint of Eq. (15) expresses the fact that options are stacked up to the amount of kilowatts that the household wishes to procure.

In order to obtain a MILP formulation of the bilevel program presented in section 4.1 , and since the producer accounts for the optimal reaction for consumers, we would like to use the optimality conditions of the consumer model as constraints for the producer model. Thanks to the linear format of the consumer model, the optimality conditions can be described as a compilation of primal and dual feasibility and strong duality conditions [5]. The dual $\left(C D_{l}\right)$ of the optimal menu 
selection problem for the consumer is given as follows:

$$
\begin{array}{ll}
\left(C D_{l}\right): \min _{\gamma_{l}} \gamma_{l} \cdot D_{l} \\
\text { s.t. } & \gamma_{l} \geq r_{i} \cdot V_{l}\left(T_{j}\right)-\pi_{i, j}, \quad i \in \mathcal{I}, j \in \mathcal{J} \\
& \gamma_{l} \geq 0
\end{array}
$$

Strong duality is expressed by Eq. (19):

$$
\gamma_{l} \cdot D_{l}=\sum_{i \in \mathcal{I}, j \in \mathcal{J}} \sigma_{l, i, j}\left(r_{i} \cdot V_{l}\left(T_{j}\right)-\pi_{i, j}\right), \quad l \in \mathcal{L}
$$

By exploiting further the particular structure of the consumer problem, we demonstrate in proposition 1 that any consumer type $l$ may as well limit its choice to a unique option out of the menu offered by the utility. This observation is an essential tool to allow the formulation of the Stackelberg equilibrium as a mixed integer linear program.

Proposition 1. There exists $\left(\tilde{\sigma}_{l, i, j}, i \in \mathcal{I}, j \in \mathcal{J}\right)$ with $\tilde{\sigma}_{l, i, j} \in\left\{0, D_{l}\right\}$ which attains the optimal objective function value.

Proof. The proof of this proposition follows the approach of [5] and is available in the electronic supplement.

\subsection{Utility}

The utility acts as the leader in the Stackelberg game, and aims at pricing the menu so as to maximize system welfare, while accounting for the optimal response of the households to the offered menu. Thus, the utility solves a unit commitment model by accounting for the individual choice of options $\sigma_{l, i, j}^{\star}$ by each consumer type, which is the optimal solution to model $\left(C P_{l}\right)$ described in section 4.2.

In addition, the utility owns a set of renewable assets. Their production is characterized by a set of scenarios. The uncertainty taken into account in the menu design program only corresponds to long-term uncertainty, such as seasonal variations, and is represented by the scenario set $\mathcal{S}$ (see section 3). Note that the additional short-term uncertainty considered in section 3 is not integrated in this model, since the menu design is a long-term problem. This optimization program is treated as a two-stage problem (menu design in the first stage, operation of the grid in the second stage). The non-negative vector $\mathbf{d}$ corresponds to the amount of power that is offered to different options under different time periods and scenarios. The optimization is carried out over a horizon $|\mathcal{T}|$. In the test case of section 6 , this horizon corresponds to 24 hours.
The menu design problem of the utility can be summarized as follows:

$$
\begin{aligned}
& (M D) \text { : } \\
& \begin{array}{c}
\max _{\substack{\mathbf{m}, \mathbf{n}, \mathbf{0}, \mathbf{d}, \mathbf{p}, \boldsymbol{\pi}, \mathbf{r}}}-\sum_{\substack{s \in \mathcal{S}, g \in \mathcal{G} \\
t \in \mathcal{T}}} P_{s} \cdot h_{g}\left(m_{g, t, s}, n_{g, t, s}, o_{g, t, s}, p_{g, t, s}\right) \\
+\sum_{\substack{i \in \mathcal{I}, l \in \mathcal{L}, j \in \mathcal{J}}} \sigma_{l, i, j}^{\star}(\mathbf{r}, \boldsymbol{\pi}) \cdot V_{l}\left(T_{j}\right) \cdot r_{i}
\end{array} \\
& \text { s.t. } f_{g}\left(m_{g, t, s}, n_{g, t, s}, o_{g, t, s}, p_{g, t, s}\right) \leq 0, \quad g \in \mathcal{G} \text {, } \\
& s \in \mathcal{S} \\
& \sum_{i \in \mathcal{I}, j \in \mathcal{J}} d_{i, j, t, s}=\sum_{g \in \mathcal{G}} p_{g, t, s}+R_{t, s}, \quad t \in \mathcal{T}, \\
& \begin{array}{r}
d_{i, j, t, s} \leq N_{j, t} \sum_{l \in \mathcal{L}} \sigma_{l, i, j}^{\star}(\mathbf{r}, \boldsymbol{\pi}), \quad i \in \mathcal{I}, \\
j \in \mathcal{J}, t \in \mathcal{T}, s \in \mathcal{S}
\end{array} \\
& r_{i} \cdot T_{j} \sum_{l \in \mathcal{L}} \sigma_{l, i, j}^{\star}(\mathbf{r}, \boldsymbol{\pi})=\sum_{s \in \mathcal{S}, t \in \mathcal{T}} P_{s} \cdot d_{i, j, t, s}, \\
& i \in \mathcal{I}, j \in \mathcal{J} \\
& d_{i, j, t, s} \geq 0, i \in \mathcal{I}, j \in \mathcal{J}, t \in \mathcal{T}, s \in \mathcal{S} \\
& p_{g, t, s} \geq 0, \quad g \in \mathcal{G}, t \in \mathcal{T}, s \in \mathcal{S} \\
& m_{g, t, s}, n_{g, t, s}, o_{g, t, s} \in\{0,1\}, g \in \mathcal{G} \text {, } \\
& t \in \mathcal{T}, s \in \mathcal{S}
\end{aligned}
$$

The goal of the utility, which is expressed in the objective function of Eq. (20), is to maximize social welfare. The first term in the objective function corresponds to the expected production cost of the utility and the second term to the consumer benefit, as estimated from the utility based on the load duration curve that is estimated in section 3 . The function $h_{g}\left(m_{g, t, s}, n_{g, t, s}, o_{g, t, s}, p_{g, t, s}\right)$ expresses the production cost of a generator, while the vector of constraints in Eq. (21) encodes linear production constraints that relate to unit commitment and the dispatch of conventional units, such as ramp rates, minimum up and down times, and so on. Power balance is expressed in constraint (22), where $R_{t, s}$ indicates the amount of renewable (system-level solar and wind) production in period $t$ under scenario s. Constraint (23) expresses the fact that a consumer type can only be served if that type is requesting power at a given interval, and if that interval is served under option $j \in \mathcal{J}$. The binary parameter $N_{j, t}$ determines whether a certain duration option $j \in \mathcal{J}$ is being served in time period $t$ of actual operations or not. Note that, given a duration option $j \in \mathcal{J}$ and a mapping from the time indexing of a load duration curve to the time indexing of actual operations, we can define this indicator. Moreover, by definition, $\sum_{t \in \mathcal{T}} N_{j, t}=T_{j}$. In other words, service option $j$ corresponds to $T_{j}$ time periods of service. Finally, constraint (24) ensures that 
an option $i \in \mathcal{I}$ receives the requested reliability $r_{i}$.

\subsection{Bilevel Formulation of Multilevel Demand Subscription Pricing}

The Stackelberg game that is described in sections 4.2 can be formulated equivalently as a mixed integer linear program, following a similar approach to [5]. Firstly, note that Proposition 1 allows us to represent the continuous variable $\sigma_{l, i, j}$ which corresponds to the subscription choice of type $l$ consumers as the following product, $\sigma_{l, i, j}=D_{l} \cdot \mu_{l, i, j}$, where $\mu_{l, i, j} \in\{0,1\}$ are binary variables.

In order to reduce the bilevel program to a single-level problem, we append the optimality conditions of the consumer model to the utility menu design program. Here, we consider the reliability $r_{i}$, price $\pi_{i, j}$ and subscription to each option as variables. Moreover, we replace the subscription variables $\sigma_{l, i, j}$ by the previously mentioned product. These optimality conditions correspond to primal feasibility (Eq. (14) and (15)), dual feasibility (Eq. (17) and (18)) and strong duality (Eq. (19)).

We now tackle the non-convex constraints resulting from the products $r_{i} \cdot \mu_{l, i, j}$ and $\pi_{i, j} \cdot \mu_{l, i, j}$. Using McCormick envelopes, we linearize these products. In particular, note that reliability is naturally within the interval $0 \leq r_{i} \leq 1$, and price is within the interval $0 \leq \pi_{i, j} \leq \Pi^{+}$, where $\Pi^{+}$corresponds to a price limit. This allows us to replace $\pi_{i, j} \cdot \mu_{l, i, j}$ by a new variable $y_{l, i, j}$, and $r_{i} \cdot \mu_{l, i, j}$ by another one $w_{l, i, j}$. Therefore, the strong duality constraint (19) for each load type $l \in \mathcal{L}$ can be rewritten as follows:

$$
\begin{aligned}
& \gamma_{l}=\sum_{i \in \mathcal{I}, j \in \mathcal{J}} w_{l, i, j} \cdot V_{l}\left(T_{j}\right)-\sum_{i \in \mathcal{I}, j \in \mathcal{J}} y_{l, i, j} \\
& 0 \leq y_{l, i, j} \leq \Pi^{+} \cdot \mu_{l, i, j}, \quad i \in \mathcal{I}, j \in \mathcal{J} \\
& y_{l, i, j} \leq \pi_{i, j}, \quad i \in \mathcal{I}, j \in \mathcal{J} \\
& y_{l, i, j} \geq \Pi^{+} \cdot \mu_{l, i, j}+\pi_{i, j}-\Pi^{+}, \quad i \in \mathcal{I}, j \in \mathcal{J} \\
& 0 \leq w_{l, i, j} \leq \mu_{l, i, j}, \quad i \in \mathcal{I}, j \in \mathcal{J} \\
& w_{l, i, j} \leq r_{i}, \quad i \in \mathcal{I}, j \in \mathcal{J} \\
& w_{l, i, j} \geq \mu_{l, i, j}+r_{i}-1, \quad i \in \mathcal{I}, j \in \mathcal{J} \\
& 0 \leq r_{i} \leq 1, \quad i \in \mathcal{I} \\
& 0 \leq \pi_{i, j} \leq \Pi^{+}, \quad i \in \mathcal{I}, j \in \mathcal{J} \\
& \mu_{l, i, j} \in\{0,1\}, \quad i \in \mathcal{I}, j \in \mathcal{J}
\end{aligned}
$$

The final mixed integer linear program is expressed as a single-level MILP as follows:

$$
\underset{\substack{\mathbf{m}, \mathbf{n}, \mathbf{o}, \mathbf{d}, \boldsymbol{\mu}, \boldsymbol{\pi}, \mathbf{r}, \mathbf{y}, \mathbf{w}}}{(M I L P):}-\sum_{\substack{s \in \mathcal{S}, g \in \mathcal{G}}} P_{s} \cdot h_{g}\left(m_{g, t, s}, n_{g, t, s}, o_{g, t, s}, p_{g, t, s}\right)
$$

$$
\begin{aligned}
& +\sum_{\substack{i \in \mathcal{I}, l \in \mathcal{L} \\
j \in \mathcal{J}}} D_{l} \cdot V_{l}\left(T_{j}\right) \cdot w_{l, i, j} \\
& \text { s.t. } f_{g}\left(m_{g, t, s}, n_{g, t, s}, o_{g, t, s}, p_{g, t, s}\right) \leq 0, g \in \mathcal{G} \text {, } \\
& s \in \mathcal{S} \text { (39) } \\
& \sum_{i \in \mathcal{I}, j \in \mathcal{J}} d_{i, j, t, s}=\sum_{g \in \mathcal{G}} p_{g, t, s}+R_{t, s}, \quad t \in \mathcal{T}, \\
& \begin{array}{r}
d_{i, j, t, s} \leq \sum_{l \in \mathcal{L}} D_{l} \cdot N_{j, t} \cdot \mu_{l, i, j}, \quad i \in \mathcal{I}, \\
j \in \mathcal{J}, t \in \mathcal{T}, s \in \mathcal{S}
\end{array} \\
& T_{j} \sum_{l \in \mathcal{L}} D_{l} \cdot w_{l, i, j}=\sum_{s \in \mathcal{S}, t \in \mathcal{T}} P_{s} d_{i, j, t, s} \\
& i \in \mathcal{I}, j \in \mathcal{J} \\
& d_{i, j, t, s} \geq 0, \quad i \in \mathcal{I}, j \in \mathcal{J}, t \in \mathcal{T} \text {, } \\
& s \in \mathcal{S}(43) \\
& p_{g, t, s} \geq 0, \quad g \in \mathcal{G}, t \in \mathcal{T}, s \in \mathcal{S} \\
& m_{g, t, s}, n_{g, t, s}, o_{g, t, s} \in\{0,1\}, g \in \mathcal{G} \text {, } \\
& t \in \mathcal{T}, s \in \mathcal{S} \\
& \sum_{i \in \mathcal{I}, j \in \mathcal{J}} \mu_{l, i, j} \leq 1, \quad l \in \mathcal{L} \\
& \gamma_{l} \geq r_{i} \cdot V_{l}\left(T_{j}\right)-\pi_{i, j}, \quad i \in \mathcal{I}, j \in \mathcal{J}, \\
& l \in \mathcal{L} \\
& \gamma_{l} \geq 0, \quad l \in \mathcal{L} \\
& (28)-(37)
\end{aligned}
$$

\section{Household Subscription}

Once the menu design program of section 4.4 is solved in order to obtain a multilevel demand subscription menu, each household must determine which specific option to procure. Thus, each household type $h \in \mathcal{H}$ solves a menu subscription problem.

Following the model of section 3, we account for short and long-term uncertainty. Long-term uncertainty corresponds to seasonal variations and is represented by the set of scenarios $\mathcal{S}$. Short-term uncertainty is comprised of the real-time production of solar panels, and the availability of each multilevel demand subscription option. Short-term uncertainty is modeled as a scenario tree. We depict short-term uncertainty by $\omega_{[t]}=\left(\omega_{[t]}^{s}, \omega_{[t]}^{c}\right) \in\left(\Omega_{[t]}^{s} \times \Omega_{[t]}^{c}\right)=\Omega_{[t]}$, where $\omega_{[t]}^{s}$ and $\omega_{[t]}^{c}$ correspond respectively to a sequence of rooftop solar production and to a sequence of ON/OFF states for each option in the multilevel demand subscription menu up to stage $t$. Note that $P_{t, \omega_{[t]}, c}$ represents the probability of observing the sequence $\omega_{[t]}$ in the scenario tree of scenario $s \in \mathcal{S}$.

The household subscription level to each option 
is represented by variable $\sigma_{h, i, j}$. This decision is not indexed by scenario, because this choice does not depend on each scenario (i.e. it remains the same over all seasons and weekdays/weekends). Daily operational decisions, represented by the following variables $(\mathbf{l s}, \mathbf{n d}, \mathbf{b d}, \mathbf{b c}, \mathbf{e})$, are indexed by $s \in \mathcal{S}$ and $\omega_{[t]} \in \Omega_{[t]}$, because long-term and short-term realizations of uncertainty will influence household operational decisions. The entire optimization of the household is modeled using the following stochastic program:

$$
\begin{aligned}
& \left(H C_{h}\right) \text { : } \\
& \begin{array}{r}
\min _{\substack{\boldsymbol{\sigma}, \mathbf{l s}, \mathbf{n d}, \mathbf{b d}, \mathbf{b c}, \mathbf{e}}} \sum_{s \in \mathcal{S}} \sum_{t \in \mathcal{T}} \sum_{\omega_{[t]} \in \Omega_{[t]}} V_{c u t} \cdot P_{s} \cdot P_{t, \omega_{[t]}^{s, c}} \cdot l s_{t, s, \omega_{[t]}} \\
+\sum_{i \in \mathcal{I}} \sum_{j \in \mathcal{J}} \pi_{i, j} \cdot \sigma_{h, i, j}
\end{array} \\
& \text { s.t. } 0 \leq b d_{t, s, \omega_{[t]}} \leq B D_{h}, \quad t \in \mathcal{T}, s \in \mathcal{S} \text {, } \\
& \omega_{[t]} \in \Omega_{[t]} \\
& 0 \leq b c_{t, s, \omega[t]} \leq B C_{h}, \quad t \in \mathcal{T}, s \in \mathcal{S}, \\
& \omega_{[t]} \in \Omega_{[t]} \\
& 0 \leq e_{t, s, \omega_{[t]}} \leq E_{h}, \quad t \in \mathcal{T}, s \in \mathcal{S}, \\
& \omega_{[t]} \in \Omega_{[t]} \\
& e_{1, s, \omega_{1}}=b c_{1, s, \omega_{1}} \cdot \eta_{h}^{c}-\frac{b d_{1, s, \omega_{1}}}{\eta_{h}^{d}}, \\
& s \in \mathcal{S}, \omega_{[t]} \in \Omega_{[t]} \\
& e_{t, s, \omega_{[t]}}=e_{t-1, s, \omega_{[t]}}-\frac{b d_{t, s, \omega_{[t]}}}{\eta_{h}^{d}} \\
& +b c_{t, s, \omega_{[t]}} \cdot \eta_{h}^{c}, \quad t \in \mathcal{T} \backslash\{1\}, \\
& s \in \mathcal{S}, \omega_{[t]} \in \Omega_{[t]} \\
& D P_{t, s, h}-l s_{t, s, \omega_{[t]}}+b c_{t, s, \omega_{[t]}}-P V_{t, s, \omega_{t}^{s}} \\
& -b d_{t, s, \omega_{[t]}}=\sum_{i \in \mathcal{I}} n d_{i, t, s, \omega_{[t]}}, \quad t \in \mathcal{T}, s \in \mathcal{S}, \\
& \omega_{[t]}=\left(\omega_{[t]}^{s}, \omega_{[t]}^{c}\right) \in\left(\Omega_{[t]}^{s} \times \Omega_{[t]}^{c}\right) \\
& n d_{i, t, s, \omega_{[t]}} \leq \sum_{j \in \mathcal{J}} \sigma_{h, i, j} \cdot 1_{\left[i, t, \omega_{t}^{c}\right]}, \quad i \in \mathcal{I}, \\
& s \in \mathcal{S},\left(\omega_{[t]}^{s}, \omega_{[t]}^{c}\right) \in \Omega_{[t]} \\
& \sum_{t \in \mathcal{T}} n d_{i, t, s, \omega_{[t]}} \leq \sum_{j \in \mathcal{J}} T_{j} \cdot \sigma_{h, i, j}, \quad i \in \mathcal{I}, \\
& t \in \mathcal{T}, s \in \mathcal{S}, \omega_{[t]} \in \Omega_{[t]} \\
& l s_{t, s, \omega_{[t]}} \geq 0, \quad t \in \mathcal{T}, s \in \mathcal{S}, \omega_{[t]} \in \Omega_{[t]}
\end{aligned}
$$

The notation and constraints of the model are similar to those in section 3. The goal of the household, depicted in Eq. (49), is to minimize the cost of load shedding along with the cost of subscribing to a particular option of the proposed menu. Constraints (50) to (54) detail the functioning of the battery in the household. The power balance constraint for the house is given by Eq. (55), where the net demand of the household drawn from the grid variable $n d_{i, t, s, \omega_{[t]}}$ is now indexed by the different reliability option of the proposed menu. Eq. (56) expresses the upper limit on net demand that the household is entitled to. The indicator variable $1_{\left[i, t, \omega_{t}^{c}\right]}$ indicates whether a certain reliability level $i$ is being served at a given stage of a sequence of outcomes or not. Constraint (57) imposes that the amount of energy consumed under a certain option cannot exceed the total energy credits that are topped up to that reliability option $i \in \mathcal{I}$.

\section{Illustration}

\subsection{Test System}

We consider the Belgian system in a forward-looking scenario of the year 2050. We work with a representative day which is split into blocks of 4 hours (i.e. 6 time stages per day).

The conventional generation fleet of the model consists of 55 units. The installed capacity of each technology follows the projected capacity of the year 2050, according to the EU 2050 reference scenario [13]. The technical specifications of the units are available from the website of the Belgian TSO Elia [14]. The installed capacity of conventional generators, which totals $15784 \mathrm{MW}$, can be broken down as follows: gas (14965 MW), oil (10 MW), biomass (542 MW), and waste $(267 \mathrm{MW})$. The long-term maintenance schedule of units is accounted for by derating the maximum capacity of the units by a certain availability ratio. The availability ratio follows the hourly profiles of 2015 [14].

Long-term uncertainty is modeled by eight representative days (scenarios $s \in \mathcal{S}$ ) corresponding to one representative weekday and one weekend day in each season of the year. For each scenario, an import profile, an availability ratio, system and household level load profiles, and wind and solar production profiles are created with 4 hours resolution. The import profiles are computed as the mean profile for each representative day, based on data gathered from year 2015 [14] which are scaled up according to the EU 2050 reference scenario [13]. In order to create system and household level inflexible load profiles, the total load profile of year 2015 [14] is split into residential, industrial and commercial load, according to Synthetic Load Profiles (SLPs) [15]. Synthetic load profiles are normalized electricity consumption time series with 15-minute 
resolution that are publicly available for the residential and non-residential sectors. The load profiles are scaled up to the year 2050 according to the EU 2050 reference scenario [13].

Two categories of residential SLPs (S21 and S22) are used for describing two categories of households. According to the data, $82 \%$ of the grid connections correspond to S21 households, and 18\% correspond to S22 households [16]. Profile S21 exhibits its consumption peak during the day, while profile S22 during the night. Renewable profiles (wind and solar production) for each season are computed as the means of historical forecasts based on data from the year 2015 [14]. These profiles are scaled up according to the projected value of the year 2050 [13].

Regarding short-term uncertainty, we create a scenario tree for each season that represents the random evolution of renewable supply in real time. Only two outcomes of solar supply are considered per time stage: high (under-forecast, indicated as $\mathrm{H}$ ) or low (over-forecast, indicated as L). The probability of under/over-forecast and the average relative error at each time stage are estimated based on the historical data of year 2015. The solar production in each scenario $s \in S$ is therefore scaled up or down based on the relative error observed at each time stage.

Finally, we consider eight different household types depending on whether these types follow profiles S21 or S22, own a battery or not, and own solar panels or not. Their characteristics are presented in Table 1. Only 25\% of the households are assumed to be equipped with solar panels, with a power rating of $2.5 \mathrm{~kW}$. Moreover, one third of these households is equipped with a large battery while one other third is equipped with a smaller battery, while one third does not own any battery. The technical specifications of household batteries are presented in Table 2.

\subsection{System-Level Load Duration Curve}

In order to create a menu of options, we approximate the system-level load duration curve using the model of section 3 . We present the resulting demand functions for the first household type in Figure 2. The demand functions of each type are then aggregated, according to the number of households in each type. We thus obtain a system-level demand function, which is generally not concave. In order to derive the concave functions $V_{l}(t)$ in Eq. 13 of problem $\left(C P_{l}\right)$, which approximate the system-level demand function, we use a least-square fit that respects the concavity of $V_{l}(t)$. The concave approximation of this system-level demand function is presented in Figure 3.

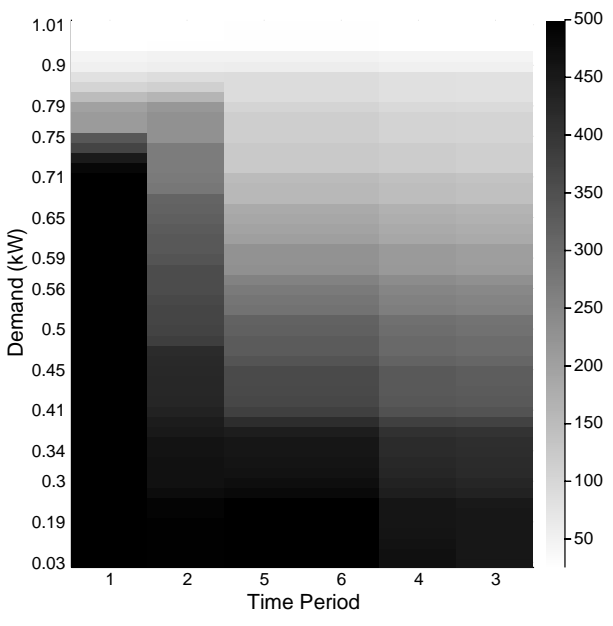

Figure 2. Demand functions for the first household type. The $x$-axis corresponds to the time period of the load duration curve, the $y$-axis represents the fuse limit. Darkness indicates valuation for an increment of fuse capacity, ranging from 0 to $500 € / \mathrm{MWh}$.

\subsection{Optimal Menus}

After computing the system-level load duration curve, we solve the MILP formulation of the Stackelberg equilibrium presented in section 4.4 in order to derive a priority service and a multilevel demand subscription menu. Indeed, since priority service pricing is a special case of multilevel demand subscription, the same MILP formulation can be used for designing a priority service menu. This optimal set of options is presented in Table 3.

In order to compare priority service and multilevel demand subscription consistently, we fix the price and reliability of multilevel demand subscription options that cover the full duration of service. By doing so, the set of options under multilevel demand subscription includes the set of options under priority service. The resulting optimal multilevel demand subscription menu is presented in Table 4.

\subsection{Household Contract Choices}

Table 5 presents the optimal subscription to the priority service menu, while table 6 presents the subscription under multilevel demand service. By comparing the energy and capacity rows of the two tables, we observe that the total subscribed capacity of each household under multilevel demand subscription is higher than under priority service. On the other hand, the subscribed energy is less. This observation is driven by the offer of options with shorter duration in multilevel 
Table 1. Characteristics of different household types.

\begin{tabular}{|c||c|c|c|c|c|c|c|c|}
\hline Household Type & Type 1 & Type 2 & Type 3 & Type 4 & Type 5 & Type 6 & Type 7 & Type 8 \\
\hline SLP Category & S21 & S22 & S21 & S22 & S21 & S22 & S21 & S22 \\
Solar Panels & Yes & Yes & Yes & Yes & Yes & Yes & No & No \\
Battery Size & Large & Large & Small & Small & No & No & No & No \\
Proportion (\%) & 6.83 & 1.5 & 6.83 & 1.5 & 6.83 & 1.5 & 61.5 & 13.5 \\
\hline
\end{tabular}

Table 2. Specifications of household batteries.

\begin{tabular}{|c||c|c|c|}
\hline $\begin{array}{c}\text { Battery } \\
\text { Type }\end{array}$ & $\begin{array}{c}\text { Capacity } \\
\text { Limit }[\mathrm{kWh}]\end{array}$ & $\begin{array}{c}\text { Power } \\
\text { Limit }[\mathrm{kW}]\end{array}$ & $\begin{array}{c}\text { Efficiency } \\
{[\%]}\end{array}$ \\
\hline Large[17] & 13.5 & 5 & 95 \\
Small[18] & 3.84 & 0.85 & 95 \\
\hline
\end{tabular}

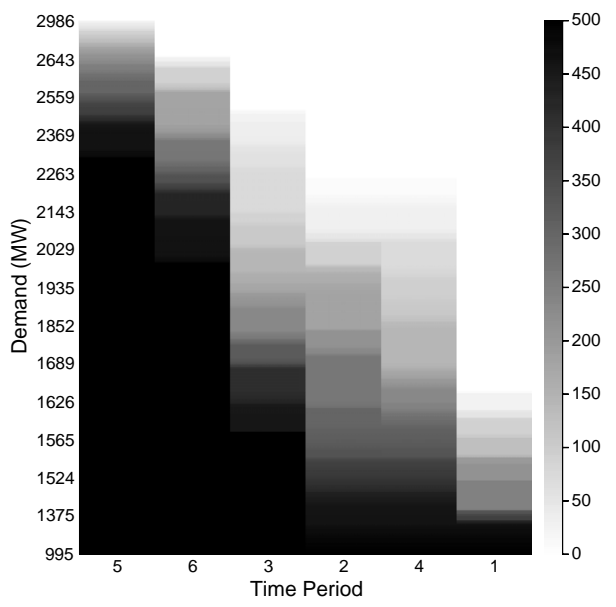

Figure 3. Concave approximation of the system-level demand function.

demand subscription. Consequently, multilevel demand subscription is not only advantageous for households, by allowing higher peak capacity when needed, but is also favorable for the producer, because the subscribed energy demand is closer to the real consumption of households. Moreover, multilevel demand subscription allows the consumer to face a lower total cost (corresponding to the sum of the service procurement cost and shortage cost) than under priority service pricing. This is due to the fact that multilevel demand subscription expresses the valuation of the consumer more accurately.

Table 3. Optimal priority service menu.

\begin{tabular}{|c|c|}
\hline Reliability [\%] & Price [€/kW-month] \\
\hline 58.5 & 26.4 \\
85.3 & 39.3 \\
100.0 & 48.5 \\
\hline
\end{tabular}

Table 4. Optimal multilevel demand subscription

\begin{tabular}{|c|c|c|}
\multicolumn{3}{c}{ menu. } \\
\hline Reliability [\%] & Duration [\%] & Price [€/kW-month] \\
\hline \multirow{3}{*}{58.5} & 33.3 & 14.9 \\
& 66.7 & 22.9 \\
& 100 & 26.4 \\
\hline \multirow{3}{*}{85.3} & 33.3 & 22.1 \\
& 66.7 & 34.1 \\
& 100 & 39.3 \\
\hline \multirow{3}{*}{100.0} & 33.3 & 27.3 \\
& 66.7 & 42.1 \\
& 100 & 48.5 \\
\hline
\end{tabular}

\section{Conclusion}

This paper focuses on the design of priority service and multilevel demand subscription as two demand response options for mobilizing flexible residential demand. We present a method for the utility to approximate the system load duration curve. We then derive a MILP formulation of the bilevel Stackelberg equilibrium for designing the two menus. Finally, we express the menu selection problem of households. We conduct a realistic case study of the Belgian market, and find that households can better match their energy consumption under multilevel demand subscription: they subscribe to less energy, and more capacity than under priority service pricing and therefore incur less total cost.

\section{Acknowledgements}

Céline Gérard is a Research Fellow (Aspirant) of the Fonds de la Recherche Scientifique - FNRS in Belgium. This research has been supported by the EPOC 2030-2050 project (Belgian energy transition funds).

\section{References}

[1] EURELECTRIC, "Dynamic pricing in electricity supply: A EURELECTRIC position paper.” http: //www.eemg-mediators.eu/downloads / dynamic_pricing_in_electricity_ supply-2017-2520-0003-01-e.pdf, Feb. 2017. 
Table 5. Priority service subscription [Energy in $\mathrm{kWh}$; payment/cost in $€ /$ month; others in $\mathrm{kW}$ ].

\begin{tabular}{|c|c|c|c|c|c|c|c|c|}
\hline Types & 1 & 2 & 3 & 4 & 5 & 6 & 7 & 8 \\
\hline Green & 0.043 & 0.04 & 0.017 & 0.021 & 0 & 0 & 0 & 0 \\
Yellow & 0.156 & 0.312 & 0.134 & 0.087 & 0.122 & 0.19 & 0.024 & 0.19 \\
Red & 1.114 & 1.311 & 1.117 & 1.555 & 1.435 & 1.787 & 1.577 & 1.787 \\
Capacity & 1.313 & 1.663 & 1.268 & 1.663 & 1.557 & 1.977 & 1.6 & 1.977 \\
Energy & 30.54 & 38.4 & 29.78 & 39.39 & 36.94 & 46.77 & 38.32 & 46.77 \\
Monthly Payment & 61.31 & 76.89 & 59.88 & 79.39 & 74.41 & 94.14 & 77.4 & 94.16 \\
Shortage Cost & 2.87 & 13.86 & 7.25 & 11.86 & 14.98 & 15.20 & 14.55 & 15.18 \\
Total Cost & 64.19 & 90.75 & 67.13 & 91.25 & 89.39 & 109.34 & 91.96 & 109.34 \\
\hline
\end{tabular}

Table 6. Multilevel demand subscription for each type of household [Energy in $\mathrm{kWh}$; payment/cost in $€ / \mathrm{month}$; others in $\mathrm{kW}$ ].

\begin{tabular}{|c|c|c|c|c|c|c|c|c|}
\hline Types & 1 & 2 & 3 & 4 & 5 & 6 & 7 & 8 \\
\hline Green-1 & 0.152 & 0.356 & 0.336 & 0.303 & 0.251 & 0.087 & 0.179 & 0.87 \\
Green-2 & 0.315 & 0 & 0 & 0 & 0 & 0 & 0 & 0 \\
Green-3 & 0 & 0 & 0 & 0 & 0 & 0 & 0 & 0 \\
Subtotal & 0.467 & 0.356 & 0.336 & 0.303 & 0.251 & 0.087 & 0.179 & 0.87 \\
\hline Yellow-1 & 0 & 0.046 & 0.063 & 0.11 & 0.229 & 0.396 & 0.155 & 0.34 \\
Yellow-2 & 0 & 0 & 0 & 0 & 0 & 0 & 0 & 0 \\
Yellow-3 & 0.104 & 0 & 0 & 0 & 0 & 0 & 0 & 0 \\
Subtotal & 0.104 & 0.046 & 0.063 & 0.11 & 0.229 & 0.396 & 0.155 & 0.34 \\
\hline Red-1 & 0 & 0.37 & 0 & 0.131 & 0.749 & 0.437 & 0.419 & 0.492 \\
Red-2 & 0 & 0 & 0 & 0.347 & 0 & 0.768 & 0.39 & 0.141 \\
Red-3 & 0.962 & 1.293 & 1.139 & 1.185 & 0.837 & 0.804 & 0.859 & 1.43 \\
Subtotal & 0.962 & 1.663 & 1.139 & 1.663 & 1.586 & 2.008 & 1.668 & 2.064 \\
\hline Capacity & 1.533 & 2.064 & 1.538 & 2.076 & 2.066 & 2.492 & 2.161 & 2.492 \\
Energy & 28.88 & 35.96 & 29.35 & 37.21 & 28.82 & 38.18 & 32.84 & 43.26 \\
Monthly Payment & 60.24 & 79.12 & 61.66 & 82.58 & 69.88 & 93.26 & 77.96 & 97.61 \\
Shortage Cost & 3.60 & 6.69 & 4.41 & 4.97 & 6.57 & 3.66 & 5.22 & 2.16 \\
Total Cost & 63.84 & 85.82 & 66.06 & 87.55 & 76.45 & 96.92 & 83.18 & 99.77 \\
\hline
\end{tabular}

[2] European Commission, "Report from the commission to the europeanparliament, the council, the european economic and social comittee and the committee of the regions: Energy prices and costs in europe - part iv: Impact of price regulation." https://ec.europa.eu/ transparency/regdoc/rep/10102/2019/ $\mathrm{EN} / \mathrm{SWD}-2019-1-\mathrm{F} 1-\mathrm{EN}-\mathrm{MAIN}-\mathrm{PART}-5 . \mathrm{PDF}$, Jan. 2019.

[3] C. Campaigne and S. S. Oren, "Firming renewable power with demand response: an end-to-end aggregator business model," Journal of Regulatory Economics, vol. 50, no. 1, pp. 1-37, 2016.

[4] K. Margellos and S. Oren, "Capacity controlled demand side management: A stochastic pricing analysis," IEEE Transactions on Power Systems, vol. 31, no. 1, pp. 706-717, 2016.

[5] Y. Mou, A. Papavasiliou, and P. Chevalier, "A bi-level optimization formulation of priority service pricing," IEEE Transactions on Power Systems, vol. 35, no. 4, pp. 2493-2505, 2020.

[6] A. Papalexopoulos, J. Beal, and S. Florek, "Precise mass-market energy demand management through stochastic distributed computing," IEEE Transactions on Smart Grid, vol. 4, no. 4, pp. 2017-2027, 2013.

[7] H.-P. Chao and R. Wilson, "Priority service: Pricing, investment, and market organization," The American Economic Review, pp. 899-916, 1987.

[8] E. Bitar and S. Low, "Deadline differentiated pricing of deferrable electric power service," in 2012 IEEE 51st IEEE Conference on Decision and Control (CDC), pp. 4991-4997, 2012.
[9] E. Bitar and Y. Xu, "Deadline differentiated pricing of deferrable electric loads," IEEE Transactions on Smart Grid, vol. 8, no. 1, pp. 13-25, 2017.

[10] A. Nayyar, M. Negrete-Pincetic, K. Poolla, and P. Varaiya, "Duration-differentiated energy services with a continuum of loads," IEEE Transactions on Control of Network Systems, vol. 3, no. 2, pp. 182-191, 2016.

[11] H.-P. Chao, S. S. Oren, S. A. Smith, and R. B. Wilson, "Multilevel demand subscription pricing for electric power," Energy economics, vol. 8, no. 4, pp. 199-217, 1986.

[12] J. Gärttner, Group formation in smart grids: Designing demand response portfolios. $\mathrm{PhD}$ thesis, Karlsruher Institut für Technologie (KIT), 2016.

[13] European Commission, "EU reference scenario 2016," October 2017.

[14] Elia, "Grid data." https://www.elia.be/en/ grid-data, Nov. 2019.

[15] Synergrid, "Synthetic load profiles (SLP)." http://www. synergrid.be/index.cfm? PageID=16896, Oct. 2017.

[16] Y. Mou, Nonlinear Pricing Schemes for Mobilizing Residential Flexibility in Power Systems. PhD thesis, Université Catholique de Louvain, 2020.

[17] Tesla, "Technical specs." https://www.tesla. com/powerwall, February 2020.

[18] Moixa, "Moixa smart battery user manual." https://www.moixa.com/wp-content/ uploads/2019/02/V4_Moixa_User_Manual_ online_UM004.pdf, February 2020. 